\title{
Ethnologies
}

\section{Réflexions autour du film The Monuments Men (2014)}

\section{Suzanne Langlois}

Volume 39, numéro 1, 2017

Géopolitique, conflits et patrimoine

Geopolitics, conflicts and heritage

URI : https://id.erudit.org/iderudit/1051055ar

DOI : https://doi.org/10.7202/1051055ar

Aller au sommaire du numéro

\section{Éditeur(s)}

Association Canadienne d'Ethnologie et de Folklore

ISSN

1481-5974 (imprimé)

1708-0401 (numérique)

Découvrir la revue

Citer cet article

Langlois, S. (2017). Réflexions autour du film The Monuments Men (2014).

Ethnologies, 39(1), 139-154. https://doi.org/10.7202/1051055ar

\section{Résumé de l'article}

The Monuments Men est un long métrage américain réalisé par George Clooney et sorti sur les écrans en février 2014. Co-scénarisé et coproduit par Clooney et son collaborateur de longue date, Grant Heslov, le film met en scène quelques membres de la section spécialisée " Monuments, Fine Arts and Archives " durant les derniers mois de la Seconde Guerre mondiale. Ce petit groupe d'engagés volontaires rattachés aux différents corps d'armée alliés avait pour mission de protéger le patrimoine culturel en temps de guerre. Les Monuments Men se révélèrent indispensables au sauvetage de vastes collections d'art spoliées par les nazis et cachées dans des dépôts en Allemagne. C'est cette course contre la montre de juin 1944 à mai 1945 que Clooney met en scène. Production hollywoodienne mélangeant les genres, le film était très attendu, mais a été largement vilipendé par la critique, bien qu'il se soit avéré un succès commercial. L'existence même d'une telle brigade spéciale en zone de guerre, le discours sur ce que représente le patrimoine culturel dans la vie des sociétés, sur la nécessité de préserver cet héritage, tous ces éléments continuent de retenir l'attention aujourd'hui. En cause, les destructions récentes d'oeuvres et de sites archéologiques majeurs dans des conflits actuels au Moyen-Orient et en Afrique, où le saccage devient une arme de guerre. En revisitant la genèse de ce film et sa mise au présent, il apparaît désormais que The Monuments Men aurait fait oeuvre utile.
Ce document est protégé par la loi sur le droit d'auteur. L'utilisation des services d’Érudit (y compris la reproduction) est assujettie à sa politique d'utilisation que vous pouvez consulter en ligne.

https://apropos.erudit.org/fr/usagers/politique-dutilisation/ 


\title{
Réflexions autour du film The Monuments Men (2014)
}

\author{
Suzanne Langlois \\ Collège Glendon de l'Université York, Toronto
}

\section{Introduction}

The Monuments Men est un long métrage réalisé par George Clooney en 2013 et sorti sur les écrans en février 2014. Coscénarisé et coproduit par Clooney et son collaborateur de longue date, Grant Heslov, le film met en scène quelques membres de la section «Monuments, Fine Arts and Archives » (ci-après MFAA), durant les derniers mois de la Seconde Guerre mondiale. Créée aux États-Unis en juin 1943 afin de protéger le patrimoine culturel en temps de guerre quand cela était possible, la petite équipe d'engagés volontaires, mieux connue sous le nom de Monuments Men, a réussi à protéger en partie le patrimoine bâti des bombardements alliés au fur et à mesure des opérations militaires, puis à localiser, sauver et restituer des milliers d'œuvres d'art saisies par les nazis. Les spoliations des collections privées appartenant aux Juifs avaient débuté en Allemagne dès l'arrivée au pouvoir de Hitler en 1933 avant de s'étendre à l'Autriche après son annexion au Reich en 1938, puis de se prolonger dans les pillages à vaste échelle de collections privées et publiques durant la guerre et l'occupation d'une grande partie de l'Europe. Ce butin a finalement compté plusieurs millions d'œuvres et documents. La mission des Monuments Men concernait aussi les édifices et les collections d'archives, le mobilier ancien et les manuscrits, mais ces domaines d'intervention ont été plus difficiles et les pertes étaient déjà immenses à leur arrivée sur les théâtres de guerre européens. En 2014, The Monuments Men a ainsi attiré l'attention 
d'un public général sur des événements qui n'étaient pas inconnus, mais dont la représentation au cinéma était déjà ancienne, ou bien dont la compréhension récente relevait d'auditoires plus localisés et spécialisés en histoire de la guerre, en cinéma documentaire ou en séries télévisées. Les réflexions présentées ici visent certains éléments internes et externes de cette production, en particulier la genèse du film en tant que sujet et représentation, puis les intersections avec le présent qui se révélaient déjà compliquées en 2013-2014 et qui restent toujours d'actualité en 2018.

\section{Les sources}

Les sources publiées sur le sujet et les références visuelles chères à George Clooney ont influencé la structure narrative et la cinématographie de The Monuments Men. Le long métrage est une adaptation du livre publié par Robert Morse Edsel en 2009 et dont la traduction française a paru dès l'année suivante. Chercheur indépendant, cet auteur américain avait d'abord fait fortune dans l'exploitation gazière et pétrolière au Texas, avant de partir vivre quelque temps à Florence à la fin des années 1990 et c'est là qu'a débuté son intérêt passionné pour le sort des œuvres d'art en temps de guerre. Investissant temps et argent dans ce projet, il a examiné de façon approfondie l'histoire de ces engagés volontaires - près de 350 hommes et femmes venant de 13 pays différents -, les champions méconnus de la protection du patrimoine culturel européen durant et après la Seconde Guerre mondiale. Leur mission s'est achevée en 1951. Edsel s'est également engagé dans la diffusion de ce sujet au moyen du film ; il a été le coproducteur du documentaire The Rape of Europa (2006), adapté du livre de l'historienne de l'art américaine et chercheure indépendante Lynn H. Nicholas, publié en 1994, ouvrage rapidement traduit en français, Le pillage de l'Europe (1995). Nicholas avait débuté ses recherches dans les années 1980. Elle est aujourd'hui experte et consultante dans des cas de restitutions d'œuvres spoliées, entre autres pour le Central Registry of Information on Looted Cultural Property 1933-1945, basé à Londres, un organisme à but non lucratif créé sous les auspices de la European Association for Jewish Studies, cette dernière étant basée à Oxford. 
Les deux maisons d'édition, Knopf pour Nicholas et Center Street (groupe Hachette) pour Edsel, visent un large lectorat intéressé, mais non spécialiste. Les éditeurs des versions françaises - Le Seuil, Lattès, Gallimard - sont également des généralistes. La traduction française de l'étude d'Edsel chez Lattès en 2010 est reprise chez Gallimard dans sa collection Folio qui paraît exactement le 27 février 2014, quelques jours à peine avant la sortie du film en France le 12 mars 2014. Cette collection de poche vise justement un accès le plus large possible non seulement aux grands textes et auteurs, mais aussi aux grands succès populaires. De plus, l'édition française ajoute dans le sous-titre le nom de Rose Valland qui n'apparaît pas dans le titre en anglais de 2009. En février et mars 2014, on parle donc à nouveau de cette résistante dans les médias, ainsi dans Paris-Match (Sprang, 24 février 2014) ou encore dans les actualités de France TV Info (Belhaoues, 8 mars 2014). Ayant rejoint les rangs de la $1{ }^{\text {ère }}$ Armée française en mai 1945, la capitaine Rose Valland fait d'ailleurs partie des «Monuments Men » présentés sur le site de la Fondation créée par Robert M. Edsel. Les informations qu'elle a pu fournir ont été cruciales pour localiser les multiples dépôts d'œuvres d'art volées et transférées sur le territoire de l'Allemagne et son rôle auprès de la Commission de récupération artistique lui a permis de suivre les œuvres durant leur rassemblement dans les points de collecte avant leur retour en France (Monuments Men Foundation, « Rose Valland »).

Finalement, il est utile de noter qu'une série télévisée en six parties, produite et diffusée au Royaume-Uni en 2014 et portant le titre de Raiders of the Lost Art, comporte un épisode intitulé Monuments Men. D'où l'importance de maintenir le titre exact du film de George Clooney - The Monuments Men.

The Monuments Men arrive donc sur les écrans après une longue maturation du sujet historique qui a mené aux débats plus récents sur la restitution des œuvres pillées par les nazis durant la Seconde Guerre mondiale. L'élément qui a probablement eu un impact important sur la façon dont George Clooney et Grant Heslov ont conçu leur scénario est le fait que Robert Edsel a lui-même déjà mis 
en place une structure narrative dans son livre sur les Monuments Men. Parmi les quelque 350 hommes et femmes qui ont participé aux opérations de la mission MFAA, Edsel a choisi de focaliser son récit autour de quelques personnages seulement. Il a même inséré dans son étude des continuités dialoguées. Ces choix semblent dictés par la volonté de rejoindre un public très large tout en respectant les sources primaires que l'auteur a consultées dans les archives américaines, les correspondances privées du temps de la guerre et des archives orales, auxquelles s'ajoutent des entretiens qu'il a réalisés avec les vétérans de cette brigade ; ce sont des sources primaires qu'il a lui-même constituées. Le contenu des dialogues n'est donc pas inventé ; pour Edsel, il s'agit plutôt d'une mise en forme dynamique des sources consultées pour faciliter la lecture et l'accès à ces informations. George Clooney et Grant Heslov ont appris cette histoire par le biais du livre d'Edsel et ils fondent leur scénario sur cette étude qui combine sources primaires et secondaires. Le réalisateur et son équipe ont fait des recherches pour les aspects cinématographiques mais le dossier de presse présentant les informations sur la production conservé aux archives de l'Academy of Motion Picture Arts and Sciences (ci-après AMPAS) ne réfère pas à d'autres éléments historiques que l'ouvrage d'Edsel nommément identifié. Clooney ne fait mention d'aucune autre démarche de recherche historique dans ses entretiens pour la presse ou la télévision. Lui, par contre, a inventé des personnages, mais il insiste sur l'histoire qui, elle, est vraie (AMPAS : 6). La durée d'écriture du scénario est d'ailleurs assez courte ; Clooney a indiqué aux journalistes allemands qui l'ont interviewé au cours du tournage à Berlin qu'il avait consacré un mois à la recherche et environ cinq mois à l'écriture (Scheiber et Lipworth 2014 : 92).

\section{La genèse visuelle}

Pour George Clooney, la genèse visuelle de son interprétation vient des classiques des films de guerre, tels que The Guns of Navarone (Les Canons de Navarone, 1961), The Great Escape (La Grande évasion, 1963) ou The Dirty Dozen (Les Douze salopards, 1967), mêlés à une approche décalée du film d'action de type « gentlemen cambrioleurs » comme Ocean's Eleven (2001) auquel l'acteur a participé dans le rôle- 
titre de Daniel Ocean. Ce mélange de film d'action (heist movie), de passages humoristiques, correspond à ce que de nombreux critiques cinématographiques mentionnent au sujet de The Monuments Men, qu'ils considèrent comme un caper movie, et qui s'avère ici un hasardeux mélange de genres qui nuit au propos du film. Les étranges références ne manquent pas : un critique allemand y voit un hybride d'Indiana Jones et du Patient anglais (Scheiber 2014 : 88). Plusieurs commentateurs s'appuient sur le solide long métrage The Train (Le Train) réalisé par John Frankenheimer en 1964 comme point de comparaison pour nourrir leur critique sévère du film de Clooney. Ce titre apparaît rarement dans les propos de Clooney : il le mentionne à Elaine Lipworth alors qu'elle l'interviewe à Berlin en 2013 (Scheiber et Lipworth 2014 : 89) et encore lorsque le journaliste Jean-Paul Chaillet rencontre le réalisateur pendant le tournage (26 août 2013) et puis à nouveau en mars 2014. Si le film de Frankenheimer était peut-être une inspiration en août 2013, il ne l'est plus en mars 2014; Clooney revient à ses références hollywoodiennes habituelles.

Oui, mais [Le Train] traitait davantage de la Résistance en France à la fin de la guerre. J'avais plutôt en tête Les Douze salopards, De l'or pour les braves, L'Express du colonel Von Ryan, Le Jour le plus long, Un pont trop loin, La Grande évasion ou La Bataille des Ardennes... Des films épiques réunissant des pléiades de stars avec du suspense et un humour un peu décalé. Je les ai revus pour essayer d'en comprendre la recette, pourquoi ils étaient toujours aussi divertissants et tenaient aussi bien la route. (Le Figaro Magazine, 6 mars 2014)

George Clooney convient qu'on ne peut refaire un film comme dans les années 1950 ; il emprunte plutôt des éléments à chacun, soit le rythme, les jeux de caméra, etc. (Scheiber et Lipworth 2014 : 90). Les termes essentiels sont toutefois là : un récit épique, des vedettes, du rythme, du suspense, de l'humour, bref, un bon divertissement. C'est ce qui dérange beaucoup la critique, mais beaucoup moins le public des salles qui ne connaît pas nécessairement Le Train et découvre avec The Monuments Men une facette moins connue et fascinante de l'histoire de la Seconde Guerre mondiale. Cependant, un public plus spécialisé a déjà eu accès au film documentaire The Rape of Europa-que Clooney a quand même pris la peine de voir - et peut-être aussi à la série télévisée The Raiders of the Lost Art. Notons 
toutefois que le discours du film Le Train était adapté du récit de Rose Valland - la résistante du Musée du Jeu de Paume - et restait centré sur la France, les œuvres étant qualifiées de patrimoine national et français, et concernait des pièces d'art moderne, des Degas, Renoir, Cézanne, etc., que les occupants souhaitaient vendre. Les tableaux étaient toujours au Musée du Jeu de Paume à l'hiver 1944 et ce n'est qu'au mois d'août 1944 qu'est formé ce « train-musée » selon le terme de Rose Valland qui décrit d'ailleurs assez brièvement cet épisode dans ses mémoires (Valland 1961 : 182-185). Le convoi ne réussit jamais à quitter la France. En cela, Le Train diffère du discours universaliste de The Monuments Men dans lequel on insiste beaucoup sur la notion de patrimoine de l'humanité malgré le fait que les œuvres montrées sont pourtant très limitées et se focalisent sur deux éléments du patrimoine religieux occidental ${ }^{1}$. Clooney avait cependant donné une vision plus large de ce qu'est le patrimoine culturel dans son entrevue à Jean-Paul Chaillet le 26 août 2013.

- Idéalement, dit-il, j'aimerais que le film, outre son caractère divertissant, sensibilise le public à l'importance de préserver notre patrimoine culturel, quelle que soit son origine... L'Amérique a commis pas mal d'erreurs dans ce domaine. Souvenez-vous de la bataille de Bagdad où l'on avait laissé détruire les musées sans intervenir. On nous en a bien fait payer

1. Il s'agit de la Madone de Bruges, œuvre sculptée par Michel-Ange vers 1501-1504 et conservée dans l'église Notre-Dame de Bruges (Belgique) et de l'Adoration de l'Agneau mystique, le polyptyque peint sur bois par les frères van Eyck, achevé en 1432, et conservé à la cathédrale Saint-Bavon de Gand (Belgique). Le dossier de presse et les informations de production (AMPAS 2014) présentent une brève chronologie de l'histoire tourmentée de l'Adoration qui est une des œuvres d'art les plus fréquemment volées au fil des siècles. Curieusement, cette chronologie s'arrête à 1935 (AMPAS : 29). Le film, quant à lui, laisse croire que les panneaux ont été directement saisis par les envahisseurs allemands alors que des responsables de la cathédrale Saint-Bavon tentaient de les mettre à l'abri en les transférant par camion. En réalité, le polyptyque avait été confié à la France par les échevins de Gand au début de la guerre et se trouvait à Pau, dans le sud-ouest de la France. Rose Valland raconte que le 3 août 1942 la nouvelle de l'enlèvement de l'Agneau mystique arrive au Louvre comme une bombe. Le télégramme de Vichy ordonnant cette remise avait été envoyé directement au conservateur du musée de Pau où se trouvait le retable et il a ainsi été rapidement remis au Dr. Buchner, directeur général des collections bavaroises (Valland 1961 : 120). Cette référence à la collaboration française du régime de Vichy avec l'Allemagne est soigneusement absente de la représentation du film au sujet des circonstances de la disparition de l'œuvre durant la guerre. 
les conséquences.

- Message reçu, conclut alors Chaillet.

Récit sobre et intense, Le Train posait toutefois de façon insistante le dilemme de risquer des vies pour un tel enjeu, celles des cheminots résistants, celles des otages, boucliers humains de la locomotive du train qui est censé sortir ces trésors nationaux de France et qui sont exécutés par les Allemands lorsque le convoi est définitivement bloqué et que les troupes d'occupation font retraite vers l'Allemagne. On est ici très loin d'Hollywood et de quelque humour décalé. Ce long métrage de 1964 doit aussi beaucoup, en termes visuels et sonores, aux noirs et blancs contrastés et aux angoissantes plages de silence de La Bataille du rail (1946) de René Clément, sa représentation réaliste des travailleurs du rail et les images spectaculaires du matériel des chemins de fer.

\section{La distribution ef la critique}

The Monuments Men profite du réseau international de distribution de SONY et de la $20^{\text {th }}$ Century Fox, d'abord en salles puis en DVD, et de la capacité à occuper les écrans du monde entier de façon massive dès février 2014. Le film était très attendu ; il avait fait l'objet de nombreux articles dans la presse générale et spécialisée durant l'année 2013 alors que les équipes étaient en tournage en Allemagne et en Grande-Bretagne. Le public sera au rendez-vous. Le film a couvert ses frais sur le seul marché américain alors que sa distribution mondiale dans 70 pays entre février et mai 2014 double ses recettes (Internet Movie Database - IMDb). The Monuments Men est un succès commercial moyen, mais un énorme échec critique. C'est bien ce qui intrigue car, malgré un épisode historique passionnant, des professionnels d'expérience - y compris l'appui de Robert Edsel -, une brochette de vedettes, un budget substantiel, l'appui d'un grand réseau mondial de distribution, tout cela n'a pas suffi pour que The Monuments Men soit un succès d'audience et considéré comme une réussite cinématographique.

La critique générale et spécialisée a amplement commenté le film dont les têtes d'affiche sont de grandes vedettes, à commencer 
par George Clooney lui-même dans le rôle-titre, et on s'entend généralement sur l'importance du sujet et la nécessité de le faire connaître le plus largement possible. Pour le reste, et c'est beaucoup, la critique a été souvent très sévère, le principal reproche étant que Clooney s'est révélé incapable de trouver le ton juste pour raconter cet extraordinaire épisode de la guerre. De « guerre d'opérette » (Morsiani 2014) à « chasse au trésor poussive »(Chaput 2014), les critiques restent perplexes quant au genre du film : drame, comédie, film de guerre, d'action, d'aventure, thriller ? Aucun de ces genres ne réussit son pari. La critique anglo-américaine est particulièrement négative et au moins deux critiques britanniques font référence à la série télévisée satirique Allô Allô (BBC, 1982-1992) pour se moquer de l'étrange accent français de Cate Blanchett (Macnab 2014 ; Kermode 2014). The Observer regrette aussi que les Britanniques soient si mal représentés alors qu'est ignorée la contribution de l'historien de l'art et médiéviste Ronald Balfour, l'un des deux membres de la MFAA à trouver la mort dans l'exercice de ses fonctions (Stummer 2014). Les ambitions de Clooney étaient ailleurs et se réduisent finalement à faire un film populaire et positif, et à avoir du plaisir à tourner avec des amis ; il était cependant bien conscient d'être passé à côté de l'essentiel.

Je me suis consacré plus de neuf mois à Monuments Men, j'ai parfois eu l'impression d'avoir eu les yeux plus gros que le ventre en m'attaquant à un projet aussi ambitieux, que c'était un challenge trop complexe et parfois même écrasant. [...] Aujourd'hui, j'estime savoir accepter et m'accommoder équitablement des succès comme des ratages. Je me suis fait une raison. (Le Figaro Magazine 6 mars 2014)

L'écart entre la réalité historique et sa transposition jugée maladroite dans un film grand public a été abondamment commentée, y compris dans des articles savants dont celui d'Elizabeth Campbell Karlsgodt (2016) qui rappelle que les historiens doivent rester vigilants et actifs au sujet d'une telle représentation, à plus forte raison puisque Sony Pictures a poussé l'ambition jusqu'à créer du matériel pédagogique à propos des événements historiques évoqués dans ce film². Absent des grandes compétitions cinématographiques, The

2. http://spi.arendtdigital.com/gh/us-movies-themonumentsmen-educationsite/ public/movies/themonumentsmen/microsite/educationsite/ 
Monuments Men a toutefois été gagnant du Heartland Film Festival (Indiana) dédié aux films qui peuvent avoir un impact positif, ou, au contraire, s'est trouvé ridiculisé par le prix du « Not-So-Obviously Worst Film » de l'Oklahoma Film Critics Circle Awards en 2014. Dans Variety, le critique Peter Bart demande à George Clooney pourquoi l'acteur-réalisateur s'entête à écrire ses propres scénarios (Variety 25 février 2014 : 26). Cette volée de bois vert n'annule cependant pas le succès commercial qui a attiré l'attention d'une nouvelle génération de spectateurs non seulement sur le sujet du film, mais plus largement sur le sort réservé au patrimoine culturel en temps de conflits et sur des zones de guerre. L'évolution des missions de la Monuments Men Foundation créée par Robert Edsel évoque cet élargissement graduel des perspectives.

Cette fondation, qui a reçu la médaille de la National Endowment for the Humanities en novembre 2007 (NEH, Scott 2007), a aujourd'hui une double mission : d'abord garder vivante l'histoire des membres de la section MFAA et leurs actions en constituant des biographies pour tous les Monuments Men ; ensuite, rester sur la trace des œuvres volées qui sont encore manquantes. Elle vérifie ainsi les collections des galeries et musées aux États-Unis et en Europe, et elle sert d'intermédiaire pour la restitution d'œuvres spoliées. Elle n'est pas seule dans ces actions, d'autres registres ayant une mission similaire. Le site "monumentsmenfoundation.org» a mis en ligne un lien et une ligne téléphonique gratuite - précisément au moment de la sortie du film de Clooney - pour encourager toute personne ayant des informations sur des objets manquants à partager ces renseignements avec elle. Après que Robert Edsel a mentionné ce numéro pendant une entrevue radio, la fondation a reçu des douzaines d'appels. On apprend ainsi que des pièces ont pu être restituées ; certaines provenaient de familles de militaires américains qui avaient acheté des œuvres durant leur service en Allemagne en 1945, tels les héritiers de Margaret Reeb en possession de deux tableaux provenant de la collection du château Friedrichshof de Kronberg au nord de Francfort, converti en club des officiers par l'armée américaine en avril 1945. D'autres avaient rapporté des « souvenirs » de leur tour de garde des dépôts d'œuvres volées par les nazis, tel cet opérateur 
radio américain rentré chez lui avec des livres rares appartenant aux collections de l'Université de Naples. Ces pièces ont été restituées à leurs propriétaires d'origine.

Tout aussi étrange retournement, le «transfert » d'œuvres prestigieuses provenant des musées de Berlin vers les États-Unis à la fin de la guerre, en totale contradiction avec la mission des Monuments Men et dénoncé comme un outrage moral par certains des professionnels sur le terrain (Karlsgodt 2016 : 399-400). Le texte de protestation rédigé par le capitaine Walter Farmer et signé par plus de 35 experts de la section MFAA est connu sous le nom de Manifeste de Wiesbaden du 7 novembre 1945. Ils n'eurent pas gain de cause. Expédiés à la National Gallery de Washington D.C., les quelque 200 tableaux de maître, des Rembrandt, Vermeer, Botticelli, Dürer, etc., ont été montrés dans plusieurs villes américaines avant d'être retournés à Wiesbaden en mai 1949 puis au musée de Dahlem dans le secteur américain de Berlin en 1955. Ces faits documentés créent des zones grises qui complexifient cette histoire. Rien de tout cela n'apparaît ou n'est mentionné dans le film, mais il faut toutefois reconnaître que l'interprétation lisse et entièrement positive de la mission américaine décrite dans The Monuments Men a rendu accessible pour un vaste public, et une autre génération, un épisode qui renseigne sur le passé et fait réfléchir sur le présent. De grands musées en ont profité pour présenter des expositions dont les dates concordent avec la sortie du film. Ainsi, la National Gallery de Washington D.C. a inauguré le 11 février 2014 son exposition «The Monuments Men and the National Gallery of Art », en concomitance avec le lancement du film. Il y a donc appropriation du sujet historique et un élargissement des lieux de diffusion vers les musées et les écoles, sans compter les relais des maisons d'édition.

\section{La mise au présent}

L'actualité du film ne fait aucun doute dans le contexte plus large des dernières années alors que les débats, expositions, restitutions sont constamment présents dans l'actualité. Prenons pour exemples les expositions d'une partie de la collection Gurlitt qui viennent 
d'ouvrir à Berne et à Bonn ${ }^{3}$ au début du mois de novembre 2017 et qui présentent des œuvres spoliées découvertes en 2012-2013 chez Cornelius Gurlitt (1932-2014), le fils d'Hildebrand Gurlitt (1895-1956), marchand et historien d'art allemand ayant profité de la vente des œuvres d'art dit dégénéré par les autorités nazies pour constituer une collection imposante de plus de 1400 œuvres dont son fils a hérité et qu'il a maintenues cachées jusqu'en 2013. Grant Heslov fait d'ailleurs référence à cet événement dans le dossier de production (AMPAS : 3). Après l'exposition «L'art dégénéré »confisqué et vendu de novembre 2017, le Kunstmuseum de Berne présentera une seconde exposition de cette collection d'avril à juillet 2018. Intitulée Collection Gurlitt, état des lieux - Les spoliations nazies et leurs conséquences, elle s'intéressera à Hildebrand Gurlitt comme acteur des spoliations nationales-socialistes (Kunstmuseum Bern). Le second exemple récent est l'annulation spectaculaire par le maire de Düsseldorf de l'exposition qui devait s'ouvrir dans sa ville en février 2018 et qui devait porter sur le catalogue de la galerie Max Stern, ce grand marchand d'art qui avait dû liquider son inventaire en 1937 avant de quitter l'Allemagne pour se réfugier d'abord en GrandeBretagne puis au Canada (Max Stern Art Restitution Project). Le contentieux porte sur la liste des œuvres.

Dans un tout autre contexte, les destructions majeures des quinze dernières années font partie des références souvent mentionnées autour de la mission des Monuments Men, plus particulièrement le pillage du musée national de l'Irak à Bagdad en avril 2003 après l'invasion américaine et à laquelle George Clooney faisait référence. Il aura fallu attendre jusqu'au 28 février 2015 pour que ce musée puisse rouvrir ses portes après avoir récupéré un tiers des 15000 pièces volées en 2003, ouverture qui se situe cependant dans un contexte tout aussi brutal et en réponse aux destructions du patrimoine culturel préislamique perpétrées par les djihadistes de Daech à Mossoul le 26 février 2015.

3. À Berne en Suisse, l'exposition «Entartete Kunst »-beschlagnahmt und verkauft du Kunstmuseum Bern, du 2 novembre 2017 au 4 mars 2018 ; à la Bundeskunsthalle de Bonn en Allemagne, l'exposition Der NS-Kunstraub und die Folgen, du 3 novembre 2017 au 11 mars 2018. 
À partir de ces éléments, le passé et le présent se rejoignent dans une même réflexion sur la place et l'importance dans la vie des individus, des sociétés et de l'humanité tout entière du patrimoine culturel dans son acception la plus large. En 2013-2014, le Krak des Chevaliers en Syrie, un site inscrit au patrimoine mondial de l'UNESCO en 2006, a subi des dommages de guerre. Au moment de ce désastre, l'historienne de l'art américaine et directrice du World Monuments Fund, Bonnie Burnham, écrivait en 2014 qu'il faudrait une nouvelle unité de Monuments Men et elle en appelait aux pays signataires de la convention de La Haye de 1954 (Convention 1954) afin de porter assistance aux sites, monuments et musées du MoyenOrient et de l'Afrique du Nord (Burnham 2014: D4). Les structures, les objets et les traditions, écrit Bonnie Burnham, forment notre héritage culturel et c'est à travers eux que se constituent nos liens à notre histoire et à notre identité ; la protection de l'héritage culturel en temps de guerre et après les conflits représente un des plus grands défis du champ culturel. Clooney, de son côté, avait expliqué que malgré l'insertion de passages plus légers et drôles, c'est la question de l'art qui est à la base de ce récit des Monuments Men ; que sans lui, la culture disparaît. Propos rapidement tempérés par Heslov qui ajoute que leur question fondamentale est : l'art vaut-il de risquer des vies? (Scheiber et Lipworth 2014 : 92)

Toutefois, face à cette interprétation large et univoque que la destruction des éléments matériels de la culture conduit à l'oubli des générations qui nous ont précédés et de leurs réalisations, à l'oblitération de la mémoire historique, un journaliste a soulevé une question à rebrousse-poil. Pour le critique de cinéma du New Yorker, David Denby (Denby 2014 : 114), le film est un échec mais il aurait pu fonctionner si Clooney avait choisi une échelle réduite, par exemple l'examen d'une obsession intellectuelle et du zèle d'experts tenant entre leurs mains des œuvres qu'ils ont étudiées toute leur vie. Clooney a plutôt choisi les grands discours sur les nazis qui ont tenté de s'approprier le passé commun de la civilisation occidentale. Cependant, poursuit Denby, beaucoup de ces œuvres devaient retourner dans des collections privées, ce qui est juste, mais convenons toutefois que ces tableaux ne faisaient pas 
partie d'un héritage commun public et partagé. Personne, semblet-il, n'a répliqué à cette remarque à contre-courant, le film ayant focalisé l'attention sur des œuvres provenant de lieux de culte ou de musées. Les acteurs eux-mêmes partagent cette perception tronquée et réfèrent aux œuvres conservées dans les musées comme étant l'essence même de la mission de sauvetage des Monuments Men (AMPAS : 4 [Cate Blanchett] et 8 [Bill Murray]) sans mention des collections privées.

\section{Conclusion}

Finalement, autour de ce long métrage à vocation populaire, et malgré une critique cinématographique sévère, il est évident qu'on a aussi beaucoup parlé de la notion de patrimoine artistique et architectural, des menaces et sauvetages en temps de guerre, de pérennité et d'appartenance culturelle, et pas seulement de la civilisation occidentale et des trésors artistiques de l'Europe. De ce point de vue, et en conjonction avec des actions culturelles entreprises en dehors du monde du cinéma, The Monuments Men aura quand même servi à rendre le sujet présent dans l'espace public et, finalement, à faire œuvre utile contre l'oubli. 


\section{Références}

Academy of Motion Picture Arts and Sciences (AMPAS), 2014, Archives de production, The Monuments Men, dossier de presse «Production information ». Margaret Herrick Library, Beverley Hills.

Bart, Peter, 2014, «"Monuments Men” Mashup Defies Clooney Mystique ». Variety $323(4): 26$

Burnham, Bonnie, 2014, "Cultural Preservation: Bring Back the Monuments Men ». Wall Street Journal, Eastern edition, 3 avril : D4.

Chaput, Luc, 2014, «The Monuments Men : chasse au trésor poussive ». Séquences $289: 54$.

Denby, David, 2014, «Art and Soul ». The New Yorker, 17 février.

Edsel, Robert Morse, 2009, The Monuments Men. Allied Heroes, Nazi Thieves, and the greatest treasure hunt in history. Nashville, Center Street. 2010, Monuments Men. Rose Valland et le commando d'experts à la recherche du plus grand trésor nazi. Paris, Lattès.

_ 2016, «Honor Your History: Celebrate Your Heroes! » Museum, mai-juin : 32-37.

Karlsgodt, Elizabeth Campbell, 2016, «What's wrong with this picture: casual disregard for history in George Clooney's The monuments men (2014) ». Historical Journal of Film, Radio and Television 36 (3) : 392-414. Kermode, Mark, 2014, «The Monuments Men: Review ». The Observer, 16 février.

Lally, Kevin, 2013, «Culture Warriors ». Film Journal International 116 (11) : 10-12.

Macnab, Geoffray, 2014, «The Monuments Men, review: George Clooney's movie is profoundly frustrating ». The Independent, 13 février.

Morsiani, Alberto, 2014, "Monuments Men di George Clooney. Una Guerra da operetta. Clooney torna all'antico ». Cineforum, Torre Boldone LIV (533) : 42-44.

Nicholas, Lynn H., 1994, The rape of Europa: the fate of Europe's treasures in the Third Reich and the Second World War. New York, Knopf.

_ 1995 , Le pillage de l'Europe. Les oeuvres d'art volées par les Nazis. Paris, Le Seuil.

Pinkerton, Nick, 2014, «The Monuments Men ». Sight and Sound 24 (4), avril : 79 .

Scheiber, Roman (texte) et Elaine Lipworth (interview), 2014, «Männer, die auf Bilder starren $»$. Ray $3:$ 86-92.

Valland, Rose, 1961, Le front de l'art. Défense des collections françaises, 1939-1945. Paris, Plon. 
Sites Internet

Belhaoues, Linda, 2014, «Rose Valland, l'une des véritables Monuments Men », 8 mars. https://culturebox.francetvinfo.fr/cinema/sorties/rosevalland-1-une-des-veritables-monuments-men-150687.

Central Registry of Information on Looted Cultural Property 1933-1945 (Londres), http://www.lootedart.com/home

Chaillet, Jean-Paul, 2013, «George Clooney et les aventuriers de l'art perdu ». Le Figaro, 26 août, http://www.lefigaro.fr/cinema/2013/08/26/03002. 20130826ARTFIG00183-george-clooney-et-les-aventuriers-de-1-artperdu.php.

Chaillet, Jean-Paul, 2014, «Interview avec Georges Clooney ». Le Figaro Magazine, 6 mars, http://www.lefigaro.fr/cinema/2014/03/06/0300220140306ARTFIG00140-georges-clooney-8220j-ai-voulu-rendrehommage-aux-heros-que-furent-les-monuments-men8221.php.

Convention pour la protection des biens culturels en cas de conflit armé (La Haye, 1954), http://unesdoc.unesco.org/images/0018/001875/187580f.pdf

European Association for Jewish Studies (Oxford), https://www. eurojewishstudies.org/

Internet Movie Database, http://www.imdb.com/title/tt2177771/ releaseinfo?ref_=ttco_sa_2

Kunstmuseum de Berne, exposition «Collection Gurlitt, état des lieux. Les spoliations nazies et leurs conséquences », avril-juillet 2018,http://www. museen-bern.ch/fr/aktuell/apercu-des-expositions/ajax-request-page/?e ventID=3689\& inst=4\& cHash=0e9adcccfd67be77719ad986ccb0fde3 .

Max Stern Art Restitution Project (Université Concordia et McGill à Montréal et Université Hébraïque de Jérusalem), https://www. concordia.ca/arts/max-stern.html.

Monuments Men Foundation for the preservation of art, https://www. monumentsmenfoundation.org/intl/fr/.

Monuments Men Foundation for the preservation of art, liste alphabétique des Monuments Men, https://www.monumentsmenfoundation.org/intl/ $\mathrm{fr} /$ the-heroes/the-monuments-men.

Monuments Men Foundation for the preservation of art, page au sujet de Rose Valland, https://www.monumentsmenfoundation.org/intl/fr/theheroes/the-monuments-men/valland-capt.-rose.

National Endowment for the Humanities, 2007, notice de Rosanne Scott au sujet du récipiendaire, Monuments Men Foundation, https://www. neh.gov/about/awards/national-humanities-medals/monuments-menfoundation-the-preservation-art.

National Gallery of Art, Washington D.C., «The Monuments Men and 
the National Gallery of Art », 11 février 2014-5 janvier 2015, https:// www.nga.gov/exhibitions/2014/monuments-men.html.

Sprang, Philippe, 2014, « Rose Valland, un chef-d'œuvre de Résistance ». Paris-Match 24 février, http://www.parismatch.com/Actu/International/ Rose-Valland-un-chef-d-oeuvre-de-Resistance-550038.

Stummer, Robin, 2014, « George Clooney's Nazi art theft film attacked for ignoring real-life British war hero ». The Observer, 19 janvier, https:// www.theguardian.com/world/2014/jan/19/george-clooney-monumentsmen-ignores-british-hero.

\section{Médiagraphie}

Monuments Men, 2014, réal. Daniel Kontur, 4 e épisode de la série Raiders of the Lost Art, Royaume-Uni, $44 \mathrm{~min}$.

The Monuments Men, 2014, réal. George Clooney, Columbia Pictures et Fox 2000 Pictures, Smokehouse Production, $119 \mathrm{~min}$.

The Rape of Europa, 2006, réal. Richard Berge, Nicole Newnham et Bonni Cohen. Venice (CA), Menemsha Films, 117 min. 